\title{
Electrically Tunable Nonlinear Refraction and Absorption in Graphene-covered SiN Waveguides
}

\author{
Koen Alexander ${ }^{1,2}$, Bart Kuyken ${ }^{1,2}$, Dries Van Thourhout ${ }^{1,2}$ \\ ${ }^{1}$ Photonics Research Group, INTEC, Ghent University-imec, Ghent B-9000, Belgium \\ ${ }^{2}$ Center for Nano- and Biophotonics (NB-Photonics), Ghent University, Ghent B-9000, Belgium \\ koen.alexander@ugent.be
}

\begin{abstract}
The real and imaginary part of the third-order nonlinearity of a gate-tunable graphene-covered SiN waveguide are measured through cross-phase and cross-amplitude modulation. A strong dependence on pump-probe detuning and Fermi energy is demonstrated. $\odot 2018$ The Author(s) OCIS codes: (130.0130) Integrated optics, (190.4380) Nonlinear optics, (160.4330) Nonlinear optical materials
\end{abstract}

\section{Introduction}

Several studies have demonstrated a strong third order nonlinear optical response in graphene [2,3]. Recently it has been shown that this response is also strongly dependent on the Fermi level $\left(E_{F}\right)$, either by measuring the four-wavemixing (FWM) or third harmonic generation (THG) response in gated graphene [1,4]. An intrinsic disadvantage of FWM and THG is that they can only be used to measure the magnitude of the third order nonlinear susceptibility $\left|\chi^{(3)}\right|$ (or conductivity $\left|\sigma^{(3)}\right|$ ). However, many potential applications require the knowledge of $\chi^{(3)}$ as a complex parameter. To assess this, we have integrated graphene on a SiN waveguide and have performed a simultaneous measurement of cross-amplitude and cross-phase modulation (XAM/XPM). We measured, for the first time to our knowledge, the complex value of the waveguide nonlinear parameter $\gamma\left(\propto \chi^{(3)} \propto i \sigma^{(3)}\right)$ as a function of gating voltage and pumpprobe detuning. These measurements uncover an intricate dependence of both the nonlinear absorption and refraction in graphene on these parameters, including strong resonances and sign changes.

\section{Experimental results}

Sample fabrication and characterization The waveguide design and fabrication were the same as in Ref. [1], Fig. 1(d) shows the cross-section and TE00 mode. Monolayer graphene was transferred to the samples by Graphenea, after which it was patterned and contacted as can be seen on the top-view image in Fig. 1(c). The structures were covered with a polymer electrolyte so that the graphene can be gated using a gate voltage $V_{G}$ [1]. The resistance over a graphene sheet $R_{D S}(L=800 \mu \mathrm{m})$ and the waveguide propagation loss $\alpha$ were measured as a function of $V_{G}$. Both measurements are plotted on Fig. 1(b). Based on this, one can estimate the relation between $V_{G}$ and $E_{F}$ in the graphene [1], the estimated $E_{F}$ is plotted on the top axis of Fig. 1(b).

(a)

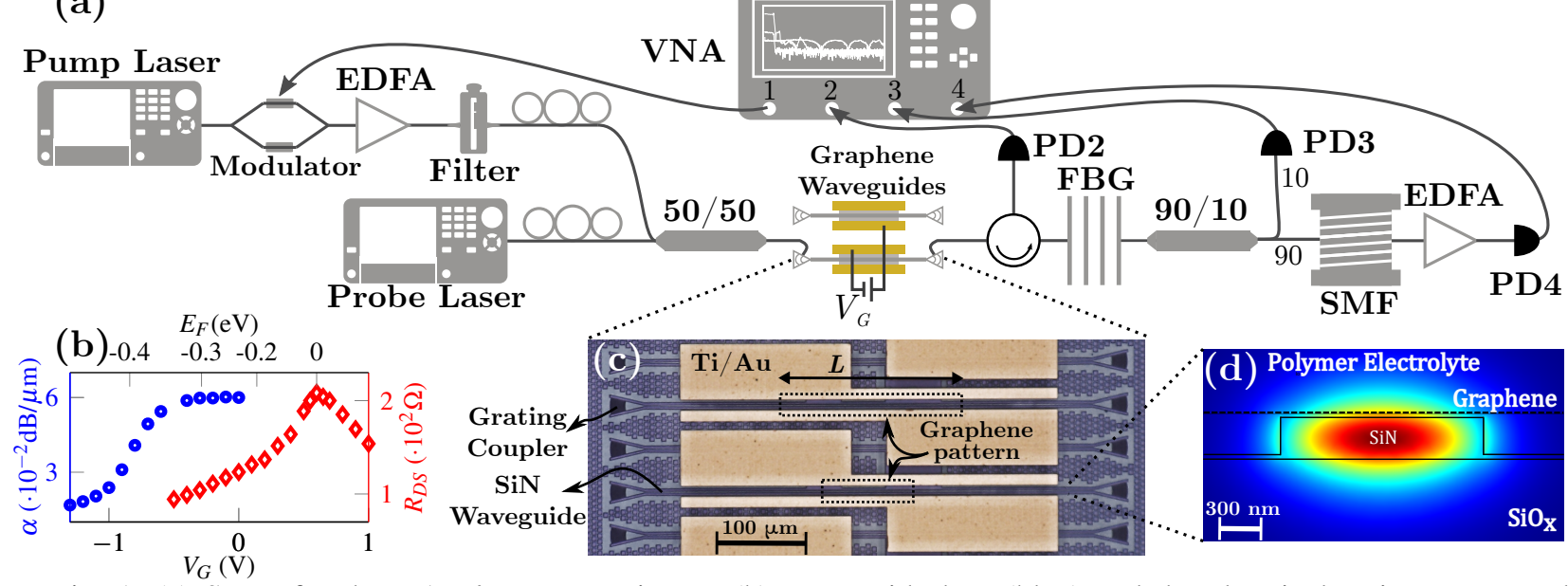

Fig. 1: (a) Setup for the XAM/XPM experiment. (b) Waveguide loss (blue) and the electrical resistance over the graphene (red) as function of $V_{G}$. (c) Top-view of the sample. (d) Cross-section of a SiN waveguide with TE00 mode. 

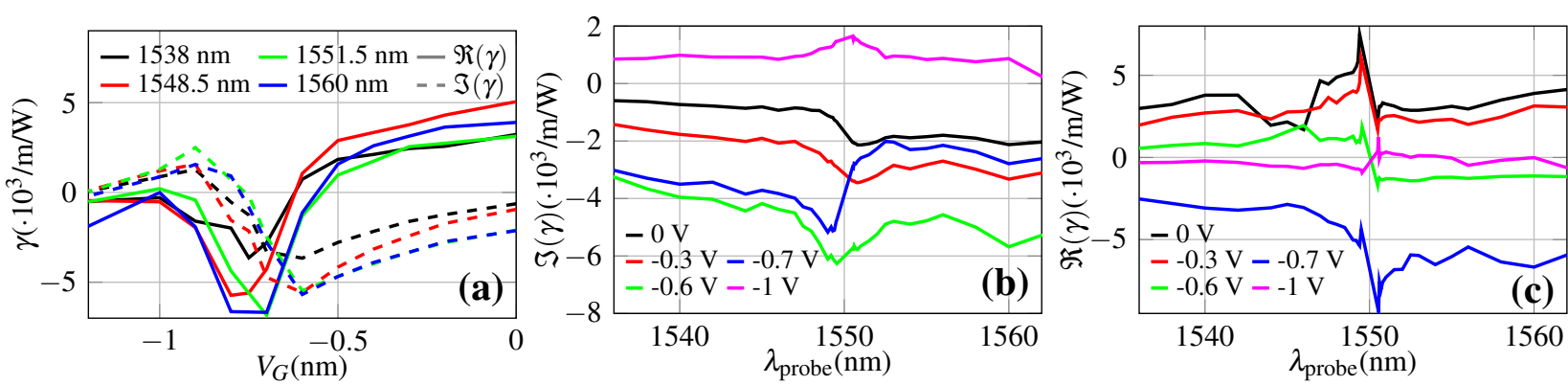

Fig. 2: Experimental results. The waveguide width and height are respectively $1400 \mathrm{~nm}$ and $330 \mathrm{~nm}$, the graphene length $50 \mu \mathrm{m}, \lambda_{\text {pump }}=1550.18 \mathrm{~nm}$. (a) $\Re \gamma$ (solid) and $\mathfrak{I} \gamma$ (dashed) as a function of $V_{G}$, for different values of $\lambda_{\text {probe }}$ (see legend). (b, c) $\mathfrak{I} \gamma$ and $\Re \gamma$ as a function of $\lambda_{\text {probe }}$, for different values of $V_{G}$ (see legend).

XAM/XPM measurement The measurement methodology was partly based on Ref. [6] and the setup is shown in Fig. 1(a). A pump laser $\left(\lambda_{\text {pump }}=1550.18 \mathrm{~nm}\right.$ ) is modulated using a vector network analyzer (VNA), combined with a weaker $\mathrm{CW}$ probe of tunable wavelength $\lambda_{\text {probe }}$ and coupled into the graphene-covered waveguide. The probe field acquires a complex phase delay $\gamma P_{\text {pump }} L_{\text {eff }}[6]$, where $L_{\text {eff }}=\left(1-e^{-\alpha L}\right) / \alpha$ and $\gamma \propto \chi^{(3)}\left(\omega_{\text {probe }}, \omega_{\text {pump }},-\omega_{\text {pump }}\right)$. XPM and XAM are thus represented by $\Re \gamma$ and $\mathfrak{I} \gamma$, respectively. After the chip, pump and probe are separated by a circulator and a fiber Bragg grating (FBG) (reflects $\lambda_{\text {pump }}$, transmits $\lambda_{\text {probe }}$ ). The pump and a fraction of the probe are sent to port 2 and 3 of the VNA, respectively. The remaining probe power is passed through $50 \mathrm{~km}$ of single mode fiber and sent to port 4 of the VNA. The voltages measured at port 2 and 3 are directly proportional to the pump and probe powers, respectively. When measuring the electrical S-parameters of the system as a function of modulation frequency $\Omega$ using the VNA, one can prove that $\lim _{\Omega \rightarrow 0} \frac{\left|S_{31}\right|}{\left|S_{21}\right|} \propto|\mathfrak{I} \gamma| L_{\text {eff }}$ and $\lim _{\Omega \rightarrow 0} \angle S_{31}-\angle S_{21}=0(=\pi)$ if $\mathfrak{I} \gamma<0(\mathfrak{I} \gamma>0)$. Furthermore, since the modulated probe propagates through a long dispersive fiber one can prove that $\left|S_{41}\right| \propto\left|\sin \left(\beta_{2} L_{\text {fiber }} \Omega^{2} / 2+\angle \gamma\right)\right|$ [6], with $L_{\text {fiber }}$ and $\beta_{2}$ the length and the group velocity dispersion of the fiber. One can thus estimate $\angle \gamma$ by fitting this relation. Hence the complex value of $\gamma$ can be derived.

Measurement results Fig. 2 summarizes a measurement of $\gamma$ of a graphene-covered waveguide $(L=50 \mu \mathrm{m}$, waveguide width $=1400 \mathrm{~nm}$ ). In Fig. 2(a), the extracted $\Re \gamma$ and $\mathfrak{I} \gamma$ are plotted as a function of $V_{G}$, for several probe wavelengths. From these curves it is clear that $\gamma$ is very dependent on $E_{F}$. $\mathfrak{I} \gamma$ is negative for low doping, $\left|E_{F}\right| \ll \hbar \omega / 2$, this corresponds to saturable absorption and is known to be strong in graphene. At high doping, $\left|E_{F}\right|>\hbar \omega / 2,|\mathfrak{I} \gamma|$ decays due to the decrease of available charge carriers. Interestingly, $\mathfrak{I} \gamma$ becomes positive right beyond the transparency point $\left(\left|E_{F}\right| \gtrsim \hbar \omega / 2\right)$, meaning that the absorption here increases with pump power. The measured $\Re \gamma$ is positive for low doping, goes through a strong resonance and becomes strongly negative around $\left|E_{F}\right| \approx \hbar \omega / 2$, after which it decays to zero. In Figs. 2(b,c), $\mathfrak{I} \gamma$ and $\Re \gamma$ are plotted as a function of $\lambda_{\text {probe }}$, for different gating voltages $V_{G} . \gamma$ is clearly dependent on wavelength, typically a resonant feature is observed around $\lambda_{\text {probe }} \approx \lambda_{\text {pump }}$.

\section{Conclusion}

For the first time to our knowledge, we simultaneously measure the nonlinear phase and amplitude response of graphene for a varying Fermi level. Both the real and imaginary part of the measured nonlinear parameter $\gamma$ of the graphene-covered waveguide are not only large in absolute terms, they are also strongly dependent on pump-probe detuning and gating voltage. The latter dependence being much more complex than what could be made out from the FWM measurement in Ref. [1]. These results can give new insight into the behavior of graphene as a nonlinear optical material and into how it can be used for tunable nonlinear applications, e.g. electrically controlled all-optical signal processing, modulating $\gamma$ for quasi phase-matched frequency conversion (as proposed in Ref. [5]), etc.

\section{References}

1. Koen Alexander et al. ACS Photonics, August 2017.

2. Evdokia Dremetsika et al. Opt. Lett., 41(14):3281-3284, 2016.

3. Euan Hendry et al. Phys. Rev. Lett., 105:097401, 2010.

4. Giancarlo Soavi et al. arXiv preprint arXiv:1710.03694, 2017.

5. Nathalie Vermeulen et al. IEEE J. Sel. Top. Quantum Electron., 22(2):347-359, 2016.

6. Jeremiah J Wathen et al. Opt. Lett., 37(22):4693-4695, 2012. 